\title{
Improving Voltage Stability in Nigerian 330kv Transmission Network Using Intelligent Solid State Var Compensator (SSVC)
}

\author{
Ngang Bassey Ngang, Bakare Kazeem \\ Department of Electrical and Electronic Engineering, Faculty of Engineering, Enugu State University of Science and Technology (ESUT), \\ Enugu, Nigeria
}

Email address:

nbngang@gmail.com (N. B. Ngang)

To cite this article:

Ngang Bassey Ngang, Bakare Kazeem. Improving Voltage Stability in Nigerian 330kv Transmission Network Using Intelligent Solid State Var Compensator (SSVC). Journal of Electrical and Electronic Engineering. Vol. 9, No. 3, 2021, pp. 60-68.

doi: $10.11648 /$ j.jeee.20210903.11

Received: May 3, 2021; Accepted: May 25, 2021; Published: May 31, 2021

\begin{abstract}
The low performance of power supply in the transmission network is as a result of voltage instability, this is overcome by improving voltage stability in Nigerian $330 \mathrm{kv}$ transmission network using intelligent solid state var compensator (SSVC). It is achieved in this procedure by running the load flow from the characterized data thereby locating the faulty buses that their per unit volts do not fall within the ranges of 0.95 through 1.05 , designing a conventional model for voltage stability in Nigerian 330KV transmission network, designing SIMULINK model for SSVC, designing a rule base that will enhance the faulty buses to attain voltage stability. Integrating the designed SIMULINK model for SSVC to designed rule base and designing a SIMULINK model for improving voltage stability in Nigerian 330KV transmission network using intelligent solid state var compensator. The results obtained were as follows: In bus1 the conventional per unit volts is 0.92 P.U.volts which did not reach the range of voltage stability of 0.95 through 1.05 P.U.volts. On the other hand, when intelligent solid state var compensator (ssvc) is incorporated in the system the per unit volts becomes 0.9779 P.U.volts thereby attaining voltage stability that enhances power supply. In bus 9 the conventional per unit volts is $0.922 \mathrm{P}$.U.volts which did not attain the voltage stability range of 0.95 through 1.05 P.U.volts. On the other hand, when intelligent solid state var compensator (ssvc) is incorporated in the system the per unit volts becomes 0.9801 P.U.volts thereby attaining voltage stability that enhances power supply.
\end{abstract}

Keywords: Improving, Voltage Stability, Nigerian 330KV, Transmission, Intelligent SSVC

\section{Introduction}

The transmission power system blackout experience in Nigeria has crippled some business outfits that solely depend on power to run their daily businesses. One of the core things that causes this intermittent power supply noticed in the country is when the per unit volts of some faulty buses not falling within the range of 0.95 through 1.05 that is meant for stability. This power instability in the country has caused the need to seek modern techniques of improving voltage stability in Nigerian $330 \mathrm{kv}$ transmission network. The use of an intelligent solid state var compensator (ssve) would be applied as would be seen in this work.

Increase in demand for electric power supply has made power system components to be stressed to their stability limits. It has negative effect on security and control.

\section{Extent of Past Work}

The analysis of the dynamic behavior of power systems for the transient stability give information about the ability of power system to sustain synchronism during and after the disturbances [1]. A power system is secured when it has the ability to withstand disturbances and return back to its normal stability state without breaking down. The state of security of a power system can be assessed by the transient stability of the system, this one of the key performance indices. In power system the small signal disturbance occurs due to changes in load and bulk transfer of power which causes generator rotor swings in presence of high gain AVR 
[2].

This has to do with the ability of power system to remain stable or revert to desired state or equilibrium when it experiences a voltage surge or system disturbances [3]. Many Techniques of assessing the unstable state of the power system is numerical integration, direct method, probabilistic technique [2] and the artificial intelligent methods such as Artificial Neural networks (ANN). [4]. The system critical Clearing Time is used to evaluate the transient Stability in response to different types of disturbance such as external faults, synchronous generator loss of excitation resulting to loss of generation and transmission line loss due to voltage surge [5]. CCT provides length of time duration a power system can be stable under system surge or disturbance condition [6]. The stability of a power system is largely dependent on its steady response to the system disturbances. A higher value of CCT shows a reliable, robust and better security system [7]. The magnitude of security level of the network is a factor in determining the level of integrity of a power system (this involves transmission capability limit and how flexible the power system becomes.) [8]. The synchronizing power coefficient of the Power system elements tied to the infinite bus bar can be improved by adequately controlling the Voltage Ampere reactive (VAr) components power triangle. Hence, Grid integrity can be enhanced by adopting a means of improving transient stability to avoid system collapse. [9].

Power system components in the transmission substation have been subjected to overloading over the years. The lack of proper maintenance planning has identified the cause of most of the system failures occurring in the national grid. Adequate maintenance planning can yield effective means of improving the transient stability without the need for constructing new transmission lines [10]. Many FACTS devices have been suggested for improving the power system operation and maintenance as proposed in the study [11]: The Static Var Compensator (SVC), Controllable Series Compensator (CSC), Phase Shifter (PS), Series Capacitors (SC), Thyristor Controlled Series Capacitors (TCSC), Unified Power Flow Controller (UPFC), Convertible Series Compensator (CSC), Inter-phase Power Flow Controller (IPFC), Static Synchronous Series Controller (SSSC), STATCOM etc. Out of all these FACTS devices, SVC seemed to be more effective for transient stability improvement when properly sized [12] and is now commonly used as an integral part of electrical power components. This paper will utilize intelligent Solid State Voltage Ampere reactive (VAr) Compensator (SSVC) to achieve a better result as could be seen at the end of the study. It is one of the modern key devices that will respond instantaneously to system changes and are fabricated manufactured from solid semiconductor devices [13]. SSVCs are usually shunt connected and are normally installed at the midpoint of the transmission line connected to a coupling or matching transformer [14]. It can improve voltage stability at the receiving end with better voltage regulation unlike the conventional methods of improving transient stability. It will eventually achieve the function of controlling its reactive power output [15]. Nigerian National grid has technical challenges due to Government protocols and foreign exchange for ordering spare parts, poor maintenance culture, and long, radial, weak and aging transmission network; Maintenance of any electrical equipment can be divided into two types namely: unscheduled maintenance and scheduled maintenance [16]. Different studies had been performed on Nigerian $330 \mathrm{kV}$ transmission network by various researchers with a view to improve the network: Recently a researcher studied and wrote on the technical losses associated with $330 \mathrm{kV}$ transmission line losses, The problem of inconsistence power supply has become the order of the day in our country Nigeria. The reasons for this is as a result of power losses in transmission network, distortion, harmonic, short circuit, burning of feeder pillars to mention a few [17].

\section{Methodology}

\subsection{Characterization of Nigerian $330 \mathrm{kv}$ Transmission Network}

Methodology used to improve the voltage stability of Nigeria 330Kk Transmission network using Intelligent Solid State VAr Compensator is outline in the following order; from step 1 to step 5. The collection and tabulation of data as shown in Table 1 is the important step used to commence the study. The collection of data from the control room electrical operators monitoring the power system parameters, was by personal interview. The control room Operation engineers were interviewed and the reason for the work was explained in order to get access to their classified data. The second step was the characterization of the network under study; load flow analysis was performed in order to locate the faulty buses whose per unit values did not fall within the range of 0.95 through 1.05 . The third step is to design a conventional model for voltage stability in Nigerian $330 \mathrm{kV}$ Transmission network. The fourth Step is the designing of a 'Rule base that makes these faulty buses to attain stability. The fifth and last stage is to integrate the designed rule to the conventional Simulink model for improving Voltage stability of the $330 \mathrm{Kv}$ Transmission network.

Table 1. 330kV Parameters for evaluating Nigerian Transmission line, Southern Nigeria, Port Harcourt-Aba.

\begin{tabular}{|c|c|c|c|c|c|c|c|c|c|c|}
\hline Bus No & Bus code & P.U & Ang Deg & Load MW & Load Mvar & Gen MW & Gen Mvar & Inject Min & Inject Max & Inject Mvar \\
\hline 1 & 1 & 0.92 & 0 & 00.0 & 0.0 & 0.0 & 0.0 & 0 & 0 & 0 \\
\hline 2 & 0 & 0.92 & 0 & 00.0 & 0.0 & 0.0 & 0.0 & 0 & 0 & 0 \\
\hline 3 & 0 & 1.0 & 0 & 150.0 & 120 & 0.0 & 0.0 & 0 & 0 & 0 \\
\hline 4 & 0 & 1.0 & 0 & 0.0 & 0.0 & 0.0 & 0.0 & 0 & 0 & 0 \\
\hline 5 & 0 & 1.0 & 0 & 120.0 & 60 & 0.0 & 0.0 & 0 & 0 & 0 \\
\hline
\end{tabular}




\begin{tabular}{lllllllllll}
\hline Bus No & Bus code & P.U & Ang Deg & Load MW & Load Mvar & Gen MW & Gen Mvar & Inject Min & Inject Max & Inject Mvar \\
\hline 6 & 0 & 0.94 & 0 & 140.0 & 90 & 0.0 & 0.0 & 0 & 0 & 0 \\
7 & 0 & 1.0 & 0 & 0.0 & 0.0 & 0.0 & 0.0 & 0 & 0 & 0 \\
8 & 0 & 1.0 & 0 & 110.0 & 90.0 & 0.0 & 0.0 & 0 & 0 & 0 \\
9 & 0 & 1.0 & 0 & 80.0 & 50.0 & 0.0 & 0.0 & 0 & 0 & 0 \\
10 & 2 & 1.025 & 0 & 0.0 & 0.0 & 200 & 0.0 & 0 & 180 & 0 \\
11 & 2 & 1.05 & 0 & 0.0 & 0.0 & 160 & 0.0 & 0 & 120 & 0 \\
\hline
\end{tabular}

To run the load flow from the characterized data thereby locating the faulty buses that their per unit volts $\operatorname{disp}(')$

basemva $=1000 ;$ accuracy $=0.0001 ;$ maxiter $=10$;

$\%$ The impedances are expressed on a 1000 MVA base.

$\%$ characterized load flow.

\begin{tabular}{|c|c|c|c|c|c|c|c|c|c|c|c|}
\hline$\%$ & Bus & Bus & $|\mathrm{V}|$ & Ang & ---Load--- & & Gen--- & Gen & Mvar & njected & \\
\hline$\%$ & No. & code & p.u. & Deg & MW & Mvar I & MW & Mvar & Min & $\operatorname{Max}$ & $\mathrm{Mv}$ \\
\hline usdata $=$ & & 1 & 0.92 & 0 & 00.0 & 0.0 & 0.0 & 0.0 & 0 & 0 & 0 \\
\hline & 2 & 0 & 0.92 & 0 & 00.0 & 0.0 & 0.0 & 0.0 & 0 & 0 & 0 \\
\hline & 3 & 0 & 1.0 & 0 & 150.0 & 120.0 & 0.0 & 0.0 & 0 & 0 & 0 \\
\hline & 4 & 0 & 1.0 & 0 & 0.0 & 0.0 & 0.0 & 0.0 & 0 & 0 & 0 \\
\hline & 5 & 0 & 1.0 & 0 & 120.0 & 60.0 & 0.0 & 0.0 & 0 & 0 & 0 \\
\hline & 6 & 0 & 0.94 & 0 & 140.0 & 90 & 0.0 & 0.0 & 0 & 0 & 0 \\
\hline & 7 & 0 & 1.0 & 0 & 0.0 & 0.0 & 0.0 & 0.0 & 0 & 0 & 0 \\
\hline & 8 & 0 & 1.0 & 0 & 110.0 & 90.0 & 0.0 & 0.0 & 0 & 0 & 0 \\
\hline & 9 & 0 & 1.0 & 0 & 80.0 & 50.0 & 0.0 & 0.0 & 0 & 0 & 0 \\
\hline & 10 & 2 & 1.025 & 0 & 0.0 & 0.0 & 200.0 & 0.0 & 0 & 180 & 0 \\
\hline & 11 & 2 & 1.05 & 0 & 0.0 & 0.0 & 160.0 & 0.0 & 0 & 120 & \\
\hline
\end{tabular}

$\begin{array}{rlllll}\% & \text { Bus } & \text { Bus } & \mathrm{R} & \mathrm{X} & 1 / 2 \mathrm{~B} \\ \% & \text { No. } & \text { No. } & \text { p.u. } & \text { p.u. } & \text { p.u. } \\ \text { linedata }=\left[\begin{array}{cccccc}1 & 2 & 0.00 & 0.06 & 0.0000 & 1 \\ 2 & 3 & 0.08 & 0.30 & 0.0004 & 1 \\ 2 & 6 & 0.12 & 0.45 & 0.0005 & 1 \\ 3 & 4 & 0.10 & 0.40 & 0.0005 & 1 \\ 3 & 6 & 0.04 & 0.40 & 0.0005 & 1 \\ 4 & 6 & 0.15 & 0.60 & 0.0008 & 1 \\ 4 & 9 & 0.18 & 0.70 & 0.0009 & 1 \\ 4 & 10 & 0.00 & 0.08 & 0.0000 & 1 \\ 5 & 7 & 0.05 & 0.43 & 0.0003 & 1 \\ 6 & 8 & 0.06 & 0.48 & 0.0000 & 1 \\ 7 & 8 & 0.06 & 0.35 & 0.0004 & 1 \\ 7 & 11 & 0.00 & 0.10 & 0.0000 & 1 \\ 8 & 9 & 0.052 & 0.48 & 0.0000 & 1\end{array}\right] ;\end{array}$

$\% \quad$ Gen. $\mathrm{Ra} \quad \mathrm{Xd}$

gendata $=\left[\begin{array}{lll}1 & 0 & 0.20\end{array}\right.$

$\begin{array}{lll}10 & 0 & 0.15\end{array}$

$\left.\begin{array}{lll}11 & 0 & 0.25\end{array}\right]$

lfybus $\quad \%$ Forms the bus admittance matrix

lfnewton $\quad \%$ Power flow solution by Newton-Raphson method

busout $\quad \%$ Prints the power flow solution on the screen

Zbus=zbuildpi(linedata, gendata, yload)\%Forms Zbus including the load

symfault(linedata, Zbus, V) \% 3-phase fault including load current 


\section{Power Flow Solution by Newton-Raphson Method \\ Maximum Power Mismatch $=8.02596 \mathrm{e}-008$ \\ No. of Iterations $=10$}

\begin{tabular}{|c|c|c|c|c|c|c|c|}
\hline \multirow{2}{*}{$\begin{array}{l}\text { Bus } \\
\text { No. }\end{array}$} & \multirow{2}{*}{$\begin{array}{l}\text { Voltage } \\
\text { Mag. }\end{array}$} & \multirow{2}{*}{$\begin{array}{l}\text { Angle } \\
\text { Degree }\end{array}$} & \multicolumn{2}{|c|}{------Load------ } & \multicolumn{2}{|c|}{---Generation--- } & \multirow{2}{*}{$\begin{array}{c}\text { Injected } \\
\text { Mvar }\end{array}$} \\
\hline & & & мU & Mvar & MU & Mvar & \\
\hline 1 & 0.920 & 0.000 & 0.000 & 0.000 & 252.346 & -11.202 & 0.000 \\
\hline 2 & 0.921 & -1.024 & 0.000 & 0.000 & 0.000 & 0.000 & 0.000 \\
\hline 3 & 0.912 & -3.802 & 150.000 & 120.000 & 0.000 & 0.000 & 0.000 \\
\hline 4 & 0.973 & -3.308 & 0.000 & 0.000 & 0.000 & 0.000 & 0.000 \\
\hline 5 & 0.944 & -9.682 & 120.000 & 60.000 & 0.000 & 0.000 & 0.000 \\
\hline 6 & 0.912 & -4.730 & 140.000 & 90.000 & 0.000 & 0.000 & 0.000 \\
\hline 7 & 0.979 & -6.665 & 0.000 & 0.000 & 0.000 & 0.000 & 0.000 \\
\hline 8 & 0.926 & -6.994 & 110.000 & 90.000 & 0.000 & 0.000 & 0.000 \\
\hline 9 & 0.922 & -6.732 & 80.000 & 50.000 & 0.000 & 0.000 & 0.000 \\
\hline 10 & 0.995 & -2.361 & 0.000 & 0.000 & 200.000 & 276.393 & 0.000 \\
\hline 11 & 1.000 & -5.728 & 0.000 & 0.000 & 160.000 & 215.626 & 0.000 \\
\hline \multicolumn{2}{|c|}{ Total } & & 600.000 & 410.000 & 612.346 & 480.817 & 0.000 \\
\hline
\end{tabular}

Figure 1. Load flow of 330KV transmission network.

In figure 1 the seven faulty buses are buses, $1,2,3,5,6,8$ and 9 with per unit volts of $0.920,0.921,0.912,0.944,0.912$, 0.926 and 0.922

\subsection{To Design a Conventional Model for Voltage Stability in Nigerian 330KV Transmission Network}

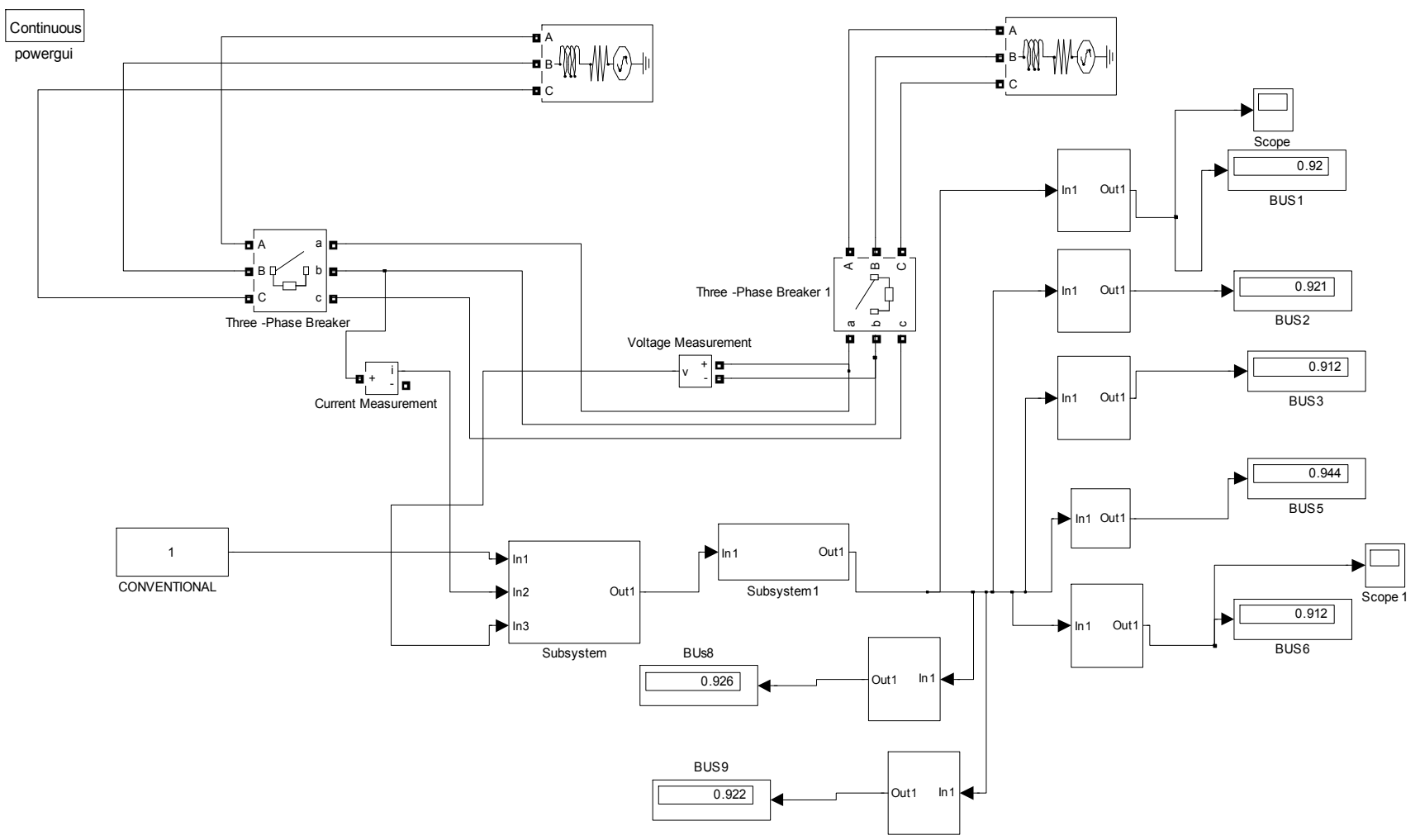

Figure 2. Designed conventional model for voltage stability in Nigerian 330KV transmission network. 


\subsection{To Design SIMULINK Model for SSVC}

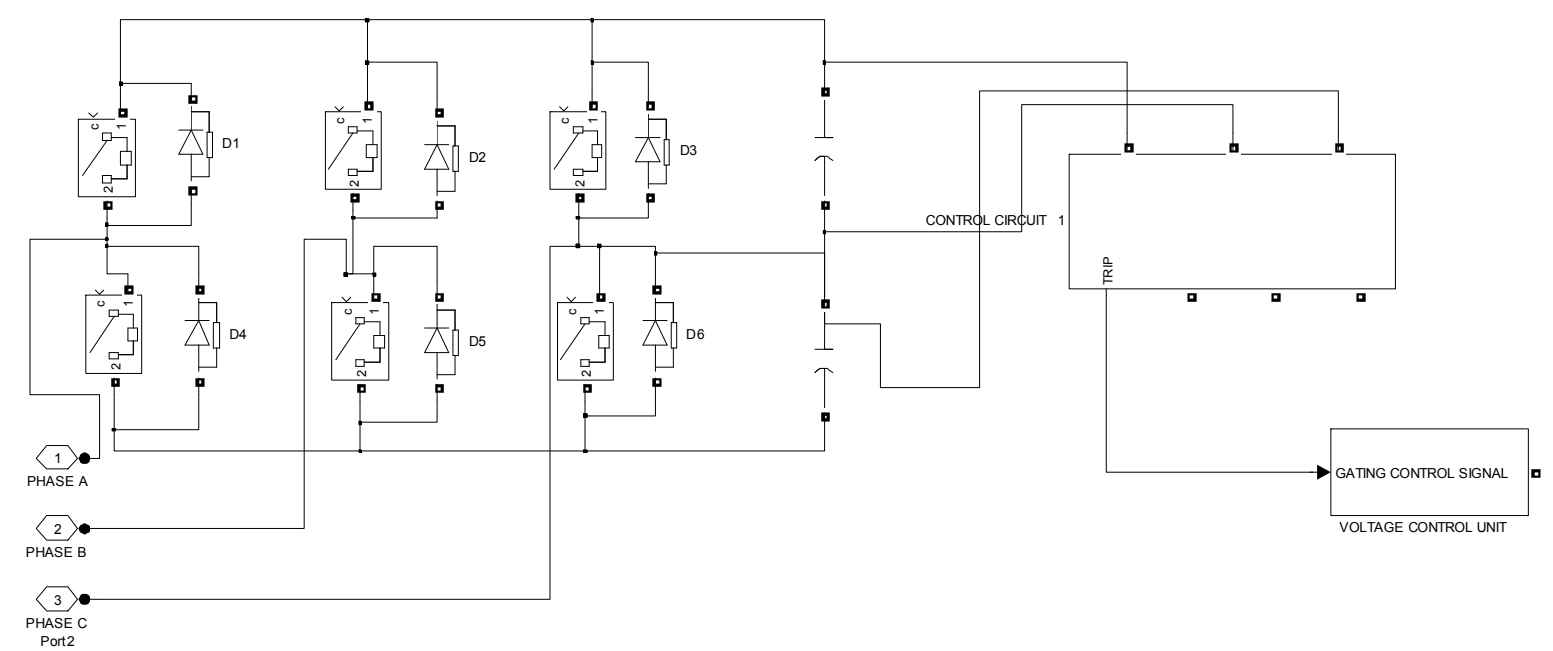

Figure 3. Designed SIMULINK model for SSVC.

\subsection{To Design a Rule Base That Will Enhance the Faulty Buses to Attain Voltage Stability}

Rule Editor: RULEBASE33

1. If (VOLTAGE is NOTMTHIN0.95THROUGH1 .05P.STABILIZE) and (POWERFACTOR is LOMNCREASE) then (RESULT is BAD) (1)

2. If (VOLTAGE is NORMAL) and (POWERFACTOR is HIGHMAINTAIN) then (RESULT is GOOD) (1)
3. If (VOLTAGE is NOTMITHINO.95THROUGH1 .05P.STABILIZE) and (POWERFACTOR is HIGHMAINTAIN) then (RESULT is BAD) (1)

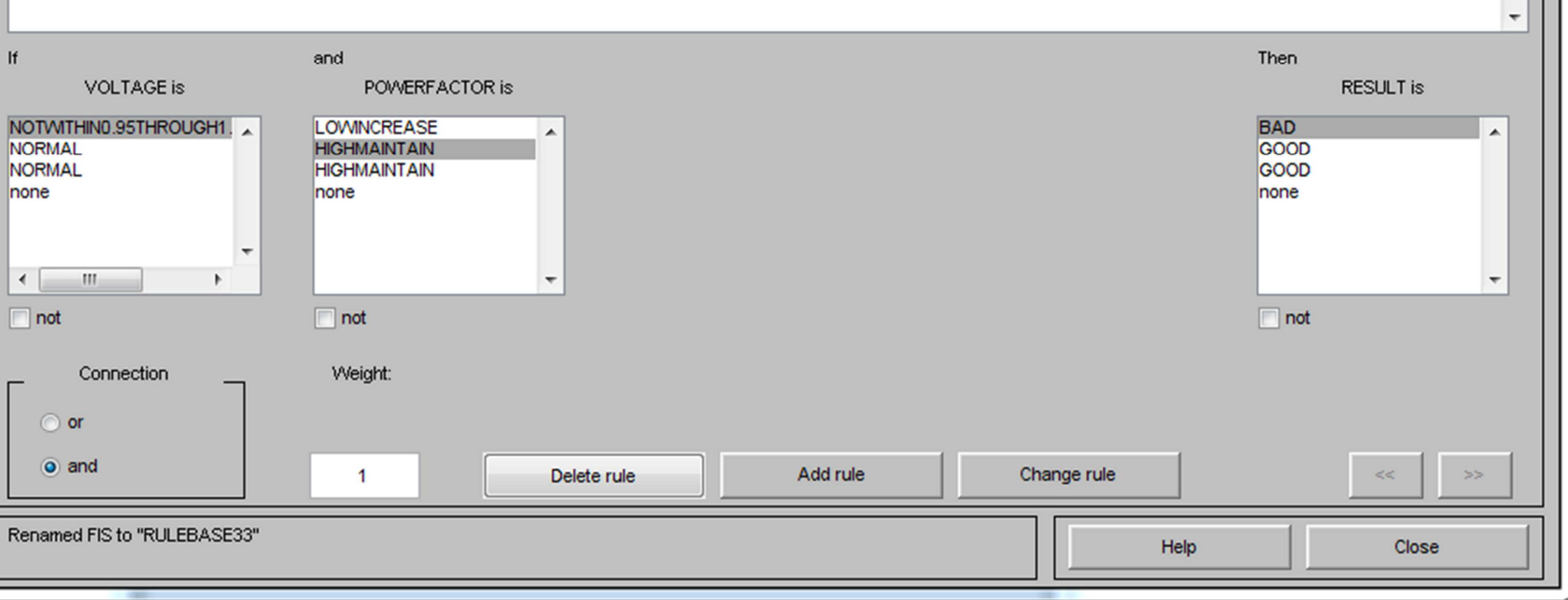

Figure 4. Designed rule base that will enhance the faulty buses to attain voltage stability. 


\subsection{To Integrate the Designed SIMULINK Model for SSVC to Designed Rule Base}

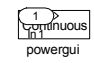

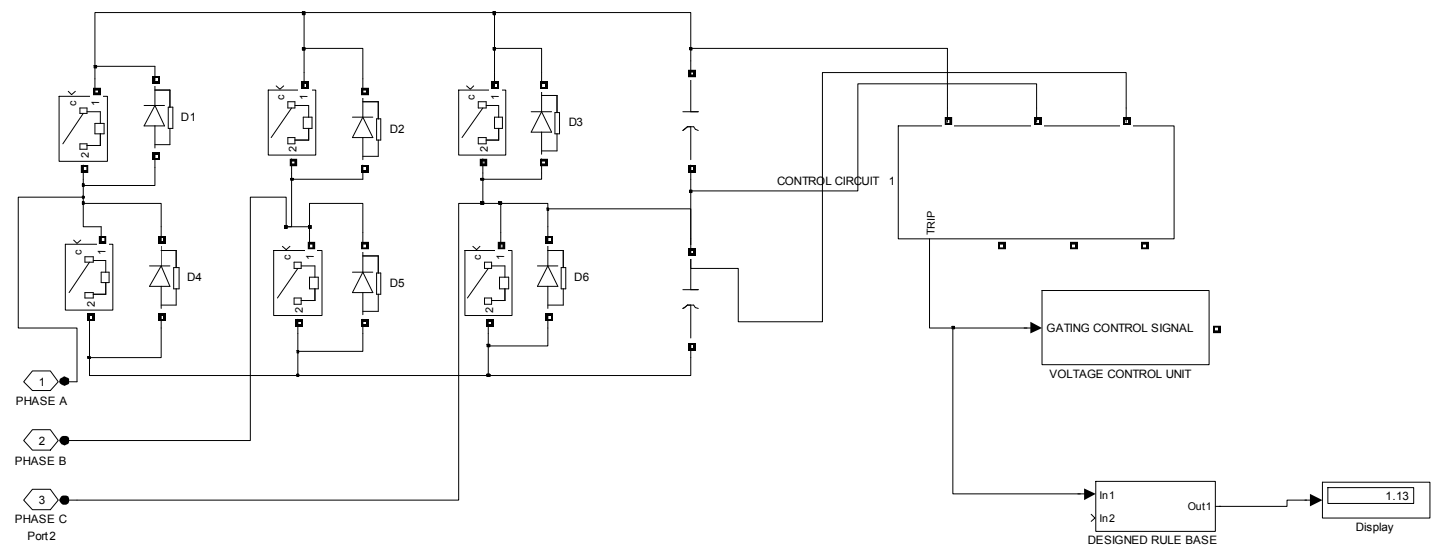

Figure 5. Integrated designed SIMULINK model for SSVC to designed rule base.

3.6. To Design a SIMULINK Model for Improving Voltage Stability in Nigerian 330KV Transmission Network Using Intelligent Solid State VA r Compensator
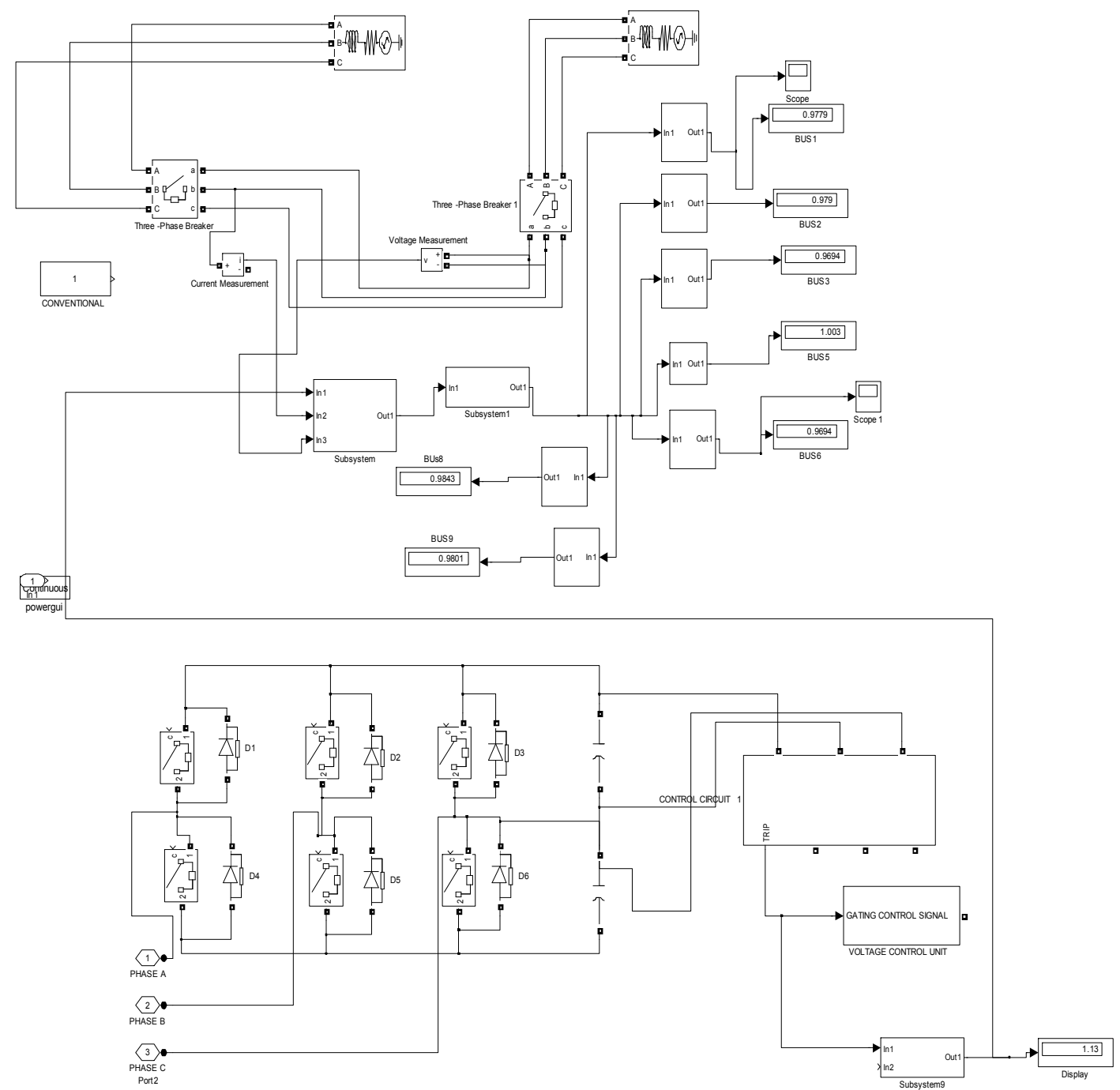

Figure 6. Designed SIMULINK model for improving voltage stability in Nigerian 330KV transmission network using intelligent solid state VAr compensator. 


\section{Results and Discussion}

In figure 1 the seven faulty buses are 1, 2, 3, 5, 6, 8 and 9 with per unit volts of $0.920,0.921,0.912,0.944,0.912,0.926$ and 0.922 ; the buses Outlined the Load flow of $330 \mathrm{KV}$ transmission network. Figure 2 displays designed conventional model for voltage stability in Nigerian $330 \mathrm{KV}$ transmission network. Figure 3 is the designed SIMULINK model for SSVC, Figure 4 shows designed rule base that will enhance the faulty buses to attain voltage stability. The rules are three in number; Figure 5 depicts integrated designed SIMULINK model for SSVC to designed rule base. Figure 6 is the designed SIMULINK model for improving voltage stability in Nigerian $330 \mathrm{KV}$ transmission network using intelligent solid state VAr compensator. Table 2 compares the conventional and intelligent solid state VAr compensator
(SSVC) in bus9 of improving voltage stability in Nigerian $330 \mathrm{kv}$ transmission network. The result obtained after simulation are as shown in figures 7 and 8. Figure 7 shows the comparison of conventional and intelligent solid state VAr compensator (SSVC) in bus1 of improving voltage stability in Nigerian $330 \mathrm{kv}$ transmission network. In figure 7 of bus 1 the conventional per unit volts is 0.92P.U.volts which did not reach the range of voltage stability of 0.95 through 1.05 P.U. volts. On the other hand, when intelligent solid state VAr compensator (SSVC) is incorporated in the system the per unit volts becomes 0.9779 P.U.volts thereby attaining voltage stability that enhances power supply. With these results, it is cleared that there is voltage stability when Intelligent Solid State Var Compensator is introduced into the system.

Table 2. Comparison between conventional and intelligent solid state VAr compensator (SSVC) in busl of improving voltage stability in Nigerian $330 \mathrm{kv}$ transmission network.

\begin{tabular}{lll}
\hline Time (s) & $\begin{array}{l}\text { Conventional voltage in bus1 of improving voltage } \\
\text { stability in Nigerian 330kv transmission network } \\
\text { (P.U.VOLTS) }\end{array}$ & $\begin{array}{l}\text { intelligent solid state VAr compensator (SSVC) in bus1 of improving } \\
\text { voltage stability in Nigerian 330kv transmission network } \\
\text { (P.U.VOLTS) }\end{array}$ \\
\hline 0 & 0 & 0 \\
1 & 0.58 & 0.6 \\
2 & 0.8 & 0.85 \\
3 & 0.87 & 0.92 \\
4 & 0.92 & 0.9779 \\
10 & 0.92 & 0.9779 \\
\hline
\end{tabular}

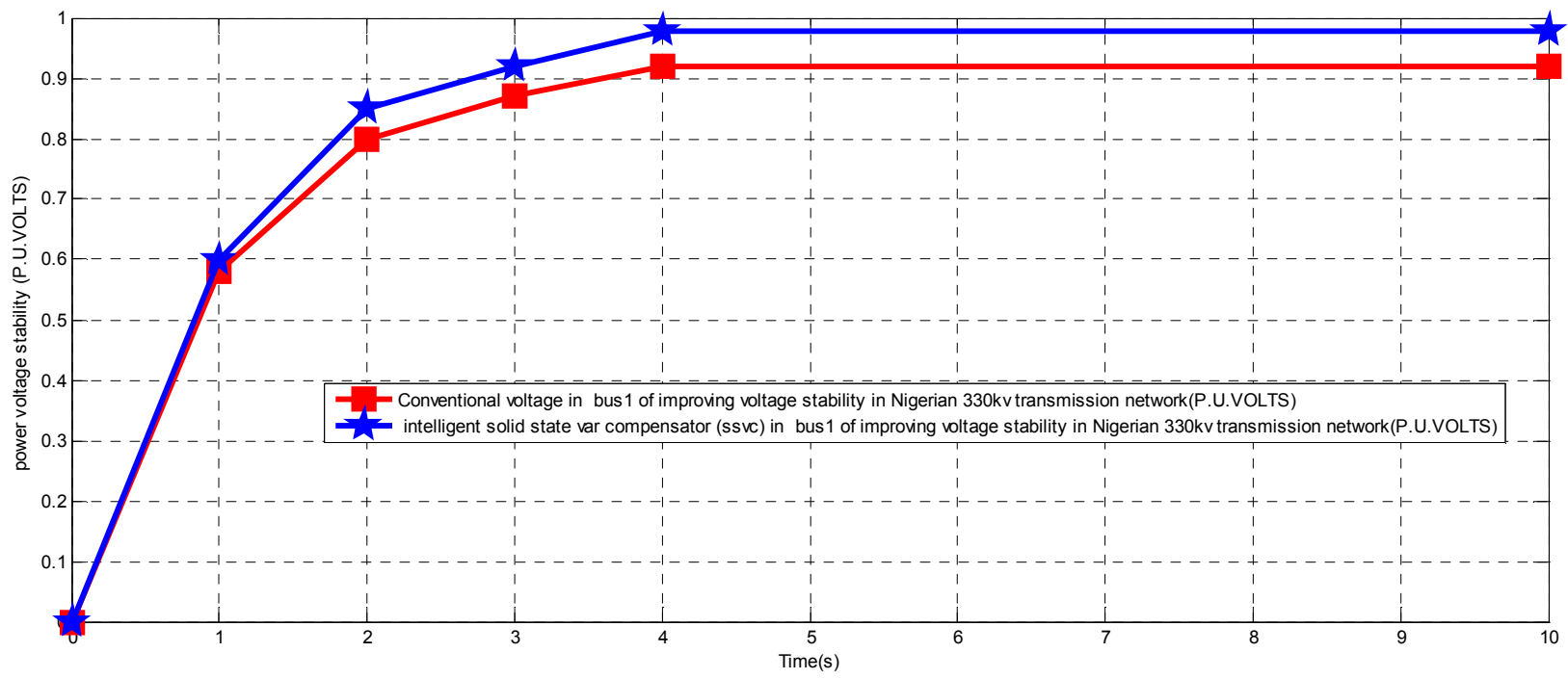

Figure 7. Comparing conventional and intelligent solid state VAR compensator (SSVC) in bus1 of improving voltage stability in Nigerian 330kv transmission network.

Table 3. Comparing conventional and intelligent solid state var compensator (ssvc) in bus 9 of improving voltage stability in Nigerian 330kv transmission network.

\begin{tabular}{lll}
\hline Time (s) & $\begin{array}{l}\text { Conventional voltage in bus9 of improving voltage stability } \\
\text { in Nigerian 330kv transmission network (P.U.VOLTS) }\end{array}$ & $\begin{array}{l}\text { intelligent solid state var compensator (ssve) in bus9 of improving } \\
\text { voltage stability in Nigerian 330kv transmission network } \\
\text { (P.U.VOLTS) }\end{array}$ \\
\hline 0 & 0 & 0 \\
1 & 0.58 & 0.6 \\
2 & 0.8 & 0.85 \\
3 & 0.88 & 0.93 \\
4 & 0.922 & 0.9801 \\
10 & 0.922 & 0.9801 \\
\hline
\end{tabular}




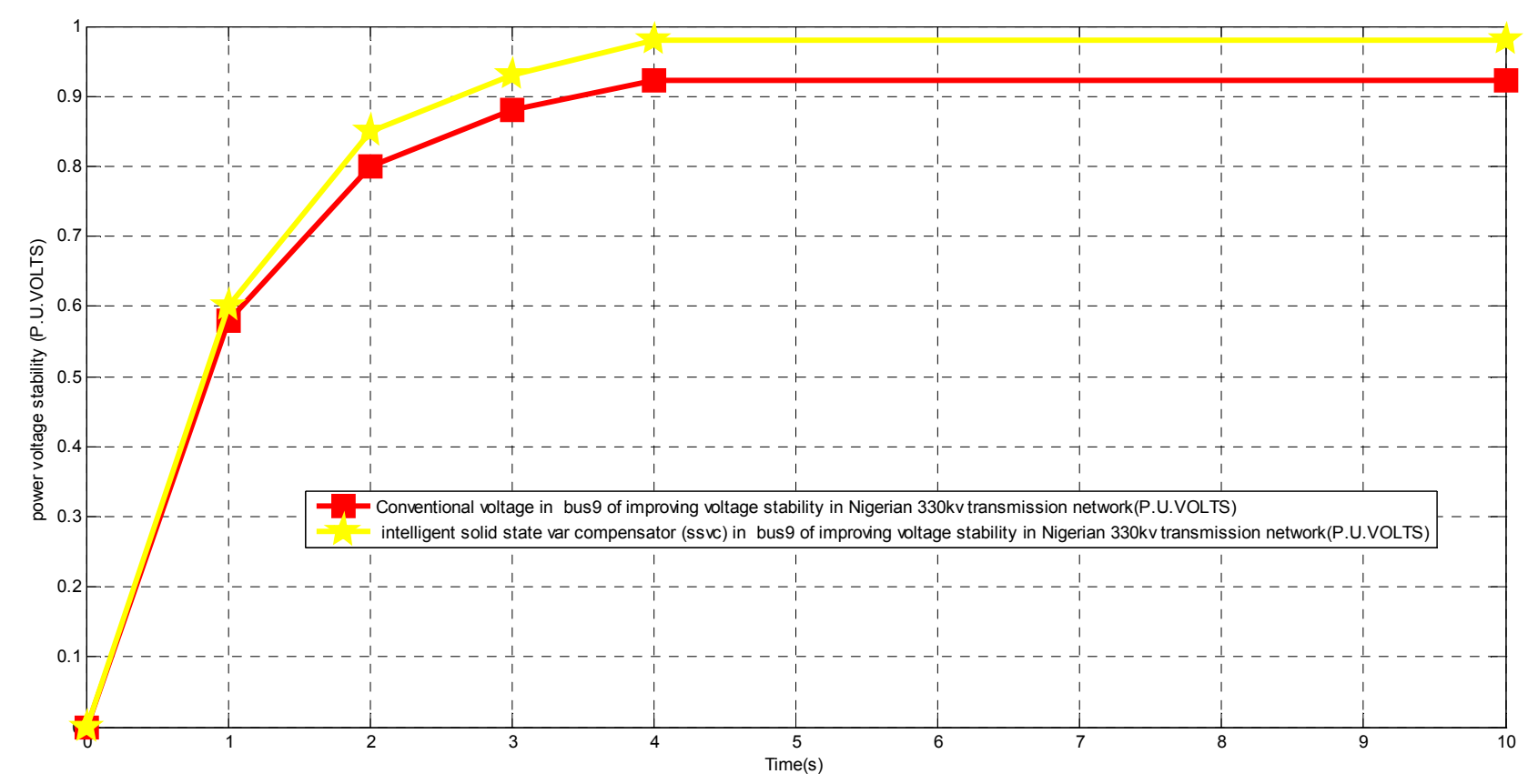

Figure 8. Comparing conventional and intelligent solid state var compensator (ssvc) in bus 9 of improving voltage stability in Nigerian 330kv transmission network.

Figure 8 shows comparing conventional and intelligent solid state var compensator (ssvc) in bus9 of improving voltage stability in Nigerian $330 \mathrm{kv}$ transmission network. In figure 8 of bus 9 the conventional per unit volts is 0.922P.U.volts which did not reach the range of voltage stability of 0.95 through 1.05 P.U. volts. On the other hand, when intelligent solid state var compensator (ssvc) is incorporated in the system the per unit volts becomes 0.9801P.U.volts thereby attaining voltage stability that enhances power supply.

\section{Conclusion}

The instability in power system in the transmission network has become a very big problem in our society. This has occurred as a result of voltage instability. This is subdued by improving voltage stability in Nigerian $330 \mathrm{kv}$ transmission network using intelligent solid state VAr compensator (SSVC). It is achieved in this procedure running the load flow from the characterized data thereby locating the faulty buses that their per unit volts do not fall within the ranges of 0.95 through 1.05 , designing a conventional model for voltage stability in Nigerian $330 \mathrm{KV}$ transmission network, designing SIMULINK model for SSVC, designing a rule base that will enhance the faulty buses to attain voltage stability. Integrating the designed SIMULINK model for SSVC to designed rule base and designing a SIMULINK model for improving voltage stability in Nigerian 330KV transmission network using intelligent solid state $\mathrm{VAr}$ compensator. The results obtained are. In figure 7 of bus 1 the conventional per unit volts is 0.92 P.U.volts which did not reach the range of voltage stability of 0.95 through 1.05 P.U.volts. On the other hand, when intelligent solid state VAr compensator (SSVC) is incorporated in the system the per unit volts becomes 0.9779 P.U.volts thereby attaining voltage stability that enhances power supply. And In figure 8 of bus 9 the conventional per unit volts is $0.922 \mathrm{P}$.U.volts which did not reach the range of voltage stability of 0.95 through 1.05 P.U.volts. On the other hand, when intelligent solid state VAr compensator (SSVC) is incorporated in the system the per unit volts becomes 0.9801 P.U.volts thereby attaining voltage stability that enhances power supply.

\section{Acknowledgements}

Appreciation goes to the Faculty of Engineering, Enugu State University of Science and Technology for the use of resource materials necessary for the completion of this research work. This work is assisted by Ngatek Global Services Limited, a private company based in Cross River State, Nigeria whose corporate objective is to support and provide funds for research works.

\section{References}

[1] Okolo C., Ezchukwu O. and Enemuo F (2020) Improving Transient Stability of the Nigerian $330 \mathrm{kV}$ transmission line System on Ajaokuta- Benin Lineusing Artificial Neural Network (ANN) BASED HIGH Voltage Voltage Direct current Method.

[2] Maity, S.; Ramya, R. A Comprehensive Review of Damping of Low Frequency Oscillations in Power Systems. Int. J. Innov. Technol. Explor. Eng. 2019, 8, 133-138.

[3] Ngang, N. B., Aneke, N. E. (2021). Enhanced Voltage Stability of the Nigerian $330 \mathrm{kV}$ Transmission Network using ANN Controller. American Journal of Applied Sciences and engineering (AJASE); Vol. 2, No. 4. DOI: 10.55393/ajase.vln2. 
[4] Chintam, J. R.; Geetha, V.; Mary, D. Optimal relocating of compensators for real-reactive power management in distributed systems. J. Electr. Eng. Technol. 2018, 13, 2145 2157.

[5] Munda, J. (2012. The impact of wind power on power system transient stability based on probabilistic weighting method. Journal of Renewable and Sustainable Energy, 4, 1-18.

[6] Ngang, N. B., Aneke, N. E. (2021), Reduction of Power system losses in Transmission Network using Optimisaion Method, International Journal of Engineering Science Invention Vol. 10, Issue 4. .

[7] Ogunjuyigbe A S O and Ayodele T R. (2015). Techno economic analysis of stand-alone hybrid energy system for nigerian telecom industry. Int. J. Renewable Energy Technology, in Press.

[8] Simeon M, Tita W S, Isaiah A A, Ajisegiri E S A, and Joseph O A. (2014). Power system's voltage stability improvement using static var compensator. International Journal of Emerging Technology and Advanced Engineering, 4, 494-501.

[9] Praveen K A, Nishant A, and Devendra M,. (2014). Improvement in power system stability with implementation of fact devices, presented at the 2nd International e-Conference on Emerging Trends in Technology, India, pp 12-15.

[10] Udhayashankar C, Thottungal R, and Yuvaraj M. (2014). Transient stability improvement in transmission system using svc with fuzzy logic control, presented at the International Conference on Advances in Electrical Engineering (ICAEE), Vellore, pp 1-4.
[11] Dhaval N T, Bhavesh B, and Vijay M, (2012), Roll of pss and svc for improving the transient stability of power system. International Journal of Engineering and Advanced Technology 1, 137-140.

[12] Aneke, N. E., Ngang, N. B. (2021) Improving the Efficacy of the Nigerian Electric Power Transmission Network Using Static Synchronous Compensator (STACOM). Journal of Information Engineering and Applications, (JIEA), Vol. 11, No. 2.

[13] Ayodele (2016). Challenges of grid integration of wind power on power system grid integrity: A review. International Journal of Renewable Energy Research, 2, 618-626.

[14] Olaiga, B. O and Olulope, P. K (2019). Voltage Stability in Nigeria Power Grid. A detailed Literature Review Vol. 2 Issue $1 \mathrm{pp} 1-10$.

[15] IEEE PES (2018). Task Force on Microgrid Stability Analysis and Modeling. Microgrid Stability Definitions, Analysis, and Modeling; Technical Report (PES-TR66); IEEE Power \& Energy Society: Piscataway, NJ, USA.

[16] Madueme, T. C. (2002). Maintenance Culture in Electrical Power Industry in Nigeria: Case Study of Afam Power Station. Nigerian Journal of Technology, Vol. 21, No. 1.

[17] Aneke, N. E and Ngang, N. B. (2021), Reduction of Power system losses in Transmission Network using Optimisaion Method, International Journal of Engineering Science Invention (IJESI) Vol. 10, Issue 4. 